\title{
The prognostic value of IncRNA SNHG6 in cancer patients
}

\author{
Haixiang Shen ${ }^{1}$, Qiwang Mo ${ }^{1,2}$ Xin Xu ${ }^{1 *}$ (D) and Ben Liu ${ }^{1 *}$
}

\begin{abstract}
Background: Although tremendous improvement has been seen in cancer diagnosis and treatment, its morbidity and mortality is still high due to lack of ideal biomarkers. An increasing number of studies have demonstrated that the expression of IncRNA small nucleolar RNA host gene 6 (SNHG6) has significantly negative correlation with various cancer prognosis. The present meta-analysis was aimed to clarify the potential of clinical application of SNHG6 in cancers.

Methods: A detailed literature review was conducted by searching through PubMed and Web of Science databases. The expression level of SNHG6, clinicopathological features and survival outcomes were extracted from eligible studies. Pooled analysis was performed with a DerSimonian-Laird random-effect model. The results were further validated through the Cancer Genome Atlas (TCGA) dataset.

Results: Five studies with a total of 487 cases were finally included in this meta-analysis. The results demonstrated that a high expression of SNHG6 was significantly associated with an increased risk of poor overall survival (OS) in cancer patients ( $H R=2.06,95 \% \mathrm{Cl} 1.56-2.73$ ). Similar results from the TCGA dataset further confirmed our findings.
\end{abstract}

Conclusions: Overexpressed SNHG6 was significantly associated with poor prognosis in various cancers. Therefore, SNHG6 may become a novel molecular target for treatment and prognostic evaluation.

Keywords: Cancer, Prognosis, Long no-coding RNA, SNHG6

\section{Background}

Cancer has become the leading cause of death globally. Over the past decades, although tremendous improvement has been achieved in its diagnosis and treatment, the prognosis is still poor, especially for advanced cancers [1]. In the United States, it was estimated that approximately $1,762,450$ cancer cases would be diagnosed and an estimated 606,880 people would die from cancer in 2019 [2]. It can impose huge financial burden on patients' families and society. Therefore, novel biomarkers are urgent to be discovered for early diagnosis, treatment and prognostic assessment.

\footnotetext{
*Correspondence: drxuxin@zju.edu.cn; drliuben@zju.edu.cn

${ }^{1}$ Department of Urology, First Affiliated Hospital, School of Medicine,

Zhejiang University, Qingchun Road 79, Hangzhou 310003, Zhejiang, China

Full list of author information is available at the end of the article
}

Among 90\% of human genome DNA that are transcribed, only $2 \%$ of them encode protein. The others which encode non-protein are named as non-coding RNAs (ncRNAs) [3, 4]. Long non-coding RNAs (lncRNAs) is a category of endogenous ncRNAs with a length of more than 200 nucleotides, which accounting for $>70 \%$ of ncRNAs [5-7]. Increasing studies have demonstrated that lncRNAs is involved in various normal cellular processes including development, differentiation and metabolism by epigenetic regulation, transcription and post-transcriptional regulation [8-11]. Recently, dysregulation of lncRNAs has been reported to be associated with oncogenesis and cancer progression [12-15], which suggested the potential of IncRNAs to be a new biomarker for early diagnosis, prognostic value and therapeutic target.

Small nucleolar RNA host gene 6 (SNHG6), also known as U87HG, is a novel lncRNA located in chromosome

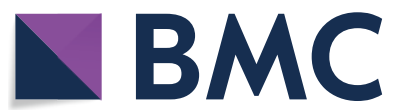

(c) The Author(s) 2020. This article is licensed under a Creative Commons Attribution 4.0 International License, which permits use, sharing, adaptation, distribution and reproduction in any medium or format, as long as you give appropriate credit to the original author(s) and the source, provide a link to the Creative Commons licence, and indicate if changes were made. The images or other third party material in this article are included in the article's Creative Commons licence, unless indicated otherwise in a credit line to the material. If material is not included in the article's Creative Commons licence and your intended use is not permitted by statutory regulation or exceeds the permitted use, you will need to obtain permission directly from the copyright holder. To view a copy of this licence, visit http://creativeco mmons.org/licenses/by/4.0/. The Creative Commons Public Domain Dedication waiver (http://creativecommons.org/publicdomain/ zero/1.0/) applies to the data made available in this article, unless otherwise stated in a credit line to the data. 
8q13.1. In 2016, Chang et al. firstly illustrated that SNHG6 was overexpressed in hepatocellular carcinoma (HCC) and promoted tumor growth and metastasis by inducing epithelial to mesenchymal transition (EMT) [16]. In recent years, accumulating evidence revealed that SNHG6 was aberrantly expression in various types of cancers and was significantly correlated with clinical stage and prognosis [16-18]. Whereas, due to small sample size in these researches, the prognostic value of SNHG6 is limited and controversial. Therefore, this meta-analysis was conducted to investigate the potential prognostic value of SNHG6 in human cancers.

\section{Methods}

\section{Literature search}

This study was performed according to the Preferred Reporting Items for Systematic Reviews and Meta-Analyses (PRISMA) statement [19]. Studies on the association between SNHG6 expression and prognosis of human cancers were identified from PubMed and Web of Science (WOS) database (up to October 30, 2019) with the following search strategy: ("small nuclear RNA host gene 6 " or "SNHG6") and ("cancer" or "carcinoma" or "neoplasm" or "tumor"). Reference lists of the identified articles and relevant reviews were manually examined for additional eligible studies. Potential eligible studies were selected by two independent authors (XX and HXS), and controversial articles were resolved by discussion and consensus.

\section{Inclusion criteria}

Studies included in this meta-analysis met all the following criteria: (i) study population was cancer patients; (ii) OS were analyzed according to SNHG6 expression pattern; (iii) multi-adjusted hazard ratios (HRs) and their 95\% confidence intervals (CIs) were provided; (iv) articles were published in English or Chinese. Studies only reported risk estimates from univariate analysis were excluded from this meta-analysis.

\section{Data extraction}

The following information was extracted from each included study by two independent authors (XX and HXS): the surname of first author, publication year, country, number of patients, age of patients, methods to determine SNHG6 expression, cut-off value, prognostic data, and adjusted variables. Any discrepancies were solved by consensus.

\section{Quality assessment}

The methodological quality of each included study was evaluated by two independent reviewers (XX and HXS) using the Newcastle-Ottawa Scale (NOS) with reasonable modifications. NOS is an eight-item instrument that focused on the characteristics of study population, study comparability, follow-up and outcome of interest. The total score of NOS is 9. A study with a score of $\geq 7$ was considered to be of high-quality.

\section{Validation by reviewing public data}

This study meets the publication guidelines provided by The Cancer Genome Atlas (TCGA). Gene Expression Profiling Interactive Analysis (GEPIA) [20] was used to verify the correlation between SNHG6 and OS and to assess the expression pattern of SNHG6 in human cancers.

\section{RNA extraction and qRT-PCR}

Total RNA was extracted from the human RCC cell lines 786-O and Caki-1, as well as one normal kidney cell line HK-2 using the RNAiso Plus (TaKaRa, Japan). Then the RNA was transcribed into cDNA using the PrimeScript RT Reagent Kit (TaKaRa, Japan). qRT-PCR assay was performed using ABI 7500 FAST Real-Time PCR System (Applied Biosystems, USA) and SYBR Green PCR Kit (Takara, China). The mRNA expression level was calculated using the $2^{-\Delta \Delta \mathrm{Ct}}$ method after normalization with $\beta$-actin. The primers involved were SNHG6 Forward 5'-ATACTTCTGCTTCGTTACCT-3'; Reverse 5'-CTC ATTTTCATCATTTGCT- $3^{\prime} ; \beta$-actin Forward $5^{\prime}$-ATC ATGAAGTGTGACGTGGAC-3'; Reverse $5^{\prime}$-GACTCG TCATACTCCTGCTTG-3'.

\section{Statistical methods}

The impact of SNHG6 expression on the OS of cancer patients was quantified by the summary HRs and their 95\% CIs. A DerSimonian-Laird random-effect model [21] was used to calculate the summary risk estimates. Existence of heterogeneity among included studies was determined using the $\mathrm{Q}$ statistic (significant level set at $0.1)$ [22]. $\mathrm{I}^{2}$ statistic was further used to assess the degree of heterogeneity (low heterogeneity: $\mathrm{I}^{2}<25 \%$; moderate heterogeneity: $\mathrm{I}^{2}=25-50 \%$; high heterogeneity: $\mathrm{I}^{2}>50 \%$ ). Sensitivity analysis was performed by sequential omission of each included study. Publication bias was assessed using a visual funnel plot. All of the statistical analyses were performed with STATA 11.0 (StataCorp, College Station, Texas USA), using two-sided P values.

\section{Results}

\section{Literature search and study characteristics}

Literature search and selection has been shown in Fig. 1. Five studies [23-27] were finally included in this metaanalysis aimed to evaluate the association between SNHG6 expression and OS of cancer patients. Three studies were performed in colorectal cancer (CRC), one 


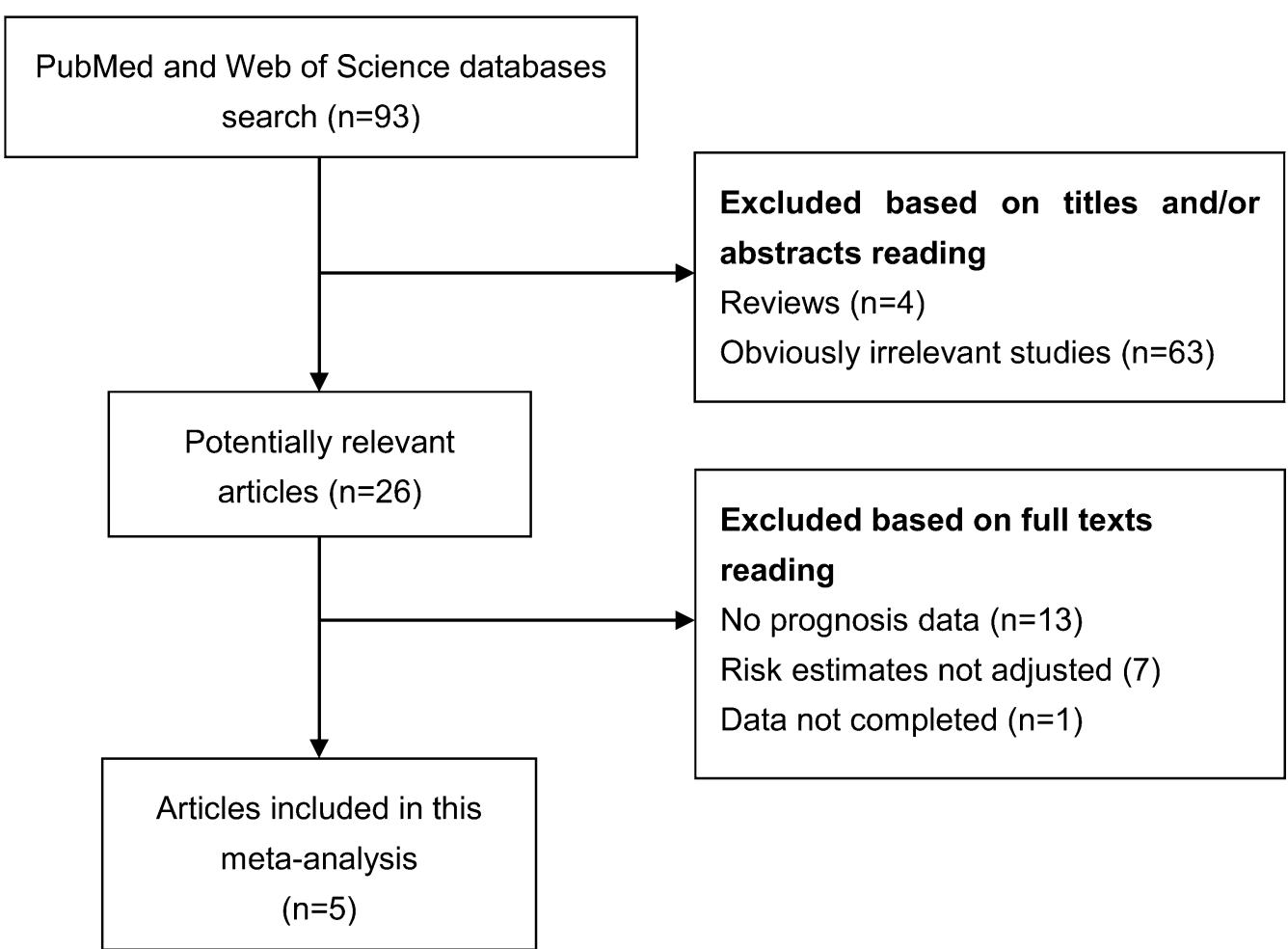

Fig. 1 The diagram shows the procedure of literature search and study selection

in renal cell carcinoma (RCC) and one in glioma. All of these studies were published between 2018 and 2019 . These studies were performed in China and involved a total of 487 cases. SNHG6 expression data was obtained by real time PCR (RT-PCR). The methodological quality, as assessed by the NOS, ranged from 8 to 9 (with a mean of 8.4). The main characteristics of each study have been summarized in Table 1.

\section{Systematic review}

Three studies performed in CRC included 74, 120, and 141 subjects, respectively. The study by Li et al. [24] indicated that SNHG6 was generally up-regulated in CRC tissues and high level of SNHG6 expression was strongly associated with advanced tumor stage $(\mathrm{P}=0.026)$ and poor prognosis $(\mathrm{P}=0.0215)$. The study by $\mathrm{Xu}$ et al. [26] reported that SNHG6 expression was an independent prognostic biomarker $(\mathrm{HR}=2.48,95 \% \mathrm{CI}=1.60-5.86$, $\mathrm{P}=0.002)$ for $\mathrm{CRC}$ in the multivariate analysis. Yu et al. [27] found high expression of SNHG6 was positively related with tumor size, advanced TNM stage, and tumor metastasis. In addition, SNHG6 was an independent prognostic factor of poor OS $(\mathrm{HR}=2.83,95 \%$ CI $1.20-8.36, \mathrm{P}=0.018)$ and $\mathrm{RFS}(\mathrm{HR}=2.07,95 \% \mathrm{CI}$ $1.17-6.20, \mathrm{P}=0.020$ ). The study by An et al. [23] reported that elevated SNHG6 was significantly associated with tumor progression and lymph node metastasis in a total of 81 cases of RCC. In addition, SNHG6 expression was

Table 1 Main characteristics of the studies included in the meta-analysis

\begin{tabular}{|c|c|c|c|c|c|c|c|}
\hline Authors & Year & Cancer & $\mathrm{HR}(95 \% \mathrm{Cl})$ & Method & No. & Follow & NOS \\
\hline An et al. & 2018 & $\mathrm{RCC}$ & $1.93(1.23-3.05)$ & RT-PCR & 81 & $80 \mathrm{~m}$ & 9 \\
\hline Li et al. & 2018 & CRC & $2.57(1.06-6.25)$ & RT-PCR & 74 & NR & 8 \\
\hline Meng et al. & 2018 & Glioma & $1.65(1.10-3.32)$ & RT-PCR & 71 & $60 \mathrm{~m}$ & 9 \\
\hline Xu et al. & 2019 & CRC & $2.48(1.60-5.86)$ & RT-PCR & 120 & NR & 8 \\
\hline Yu et al. & 2019 & CRC & $2.83(1.20-8.36)$ & RT-PCR & 141 & NR & 8 \\
\hline
\end{tabular}

$R C C$ renal cell carcinoma, $C R C$ colorectal cancer, $H R$ hazard ratio, 95\% Cl 95\% confidence interval, NR no report 
associated with overall prognosis in RCC. Meng et al. [25] investigated the role of SNHG6 in glioma and found that the expression of SNHG6 was negatively associated with the $\mathrm{OS}(\mathrm{HR}=1.65 ; 95 \%$ CI $1.10-3.32 ; \mathrm{P}=0.0076)$.

\section{Overall analysis}

The HRs for each included study and for the combination of all studies are shown in Fig. 2. A high expression of SNHG6 was significantly associated with an increased risk of poor OS ( $\mathrm{HR}=2.06,95 \% \mathrm{CI} 1.56-2.73)$. There was no obvious heterogeneity among the studies $\left(\mathrm{I}^{2}=0.0 \%\right.$; $\mathrm{P}=0.797)$.

\section{Sensitivity analysis and publication bias}

In order to assess the stability of the pooled risk estimate of the association between SNHG6 expression and OS, sensitivity analysis was performed by omitting each included study in turn. As shown in Fig. 3, the pooled result was not dominated by any single study. There was no evidence of publication bias with a visual funnel plot (Fig. 4).

\section{SNHG6 and OS of CRC}

Three studies reported data on the relationship between SNHG6 expression and OS of CRC. The summary data based on these studies indicated that SNHG6 status was significantly associated with the OS with a combined HR of 2.58 (95\% CI 1.63-4.09). No obvious heterogeneity was observed across studies $\left(\mathrm{I}^{2}=0.0 \%, \mathrm{P}=0.976\right)$.

\section{Validation of the SNHG6 expression in RCC cells}

The mRNA expression of SNHG6 in two RCC cell lines (786-O and Caki-1) and one normal kidney cell line (HK2) was detected using qRT-PCR. As a result, the expression of SNHG6 in RCCs was significantly upregulated compared with that in HK-2 cells (Additional file 1: Figure S1).

\section{Validation of the results in TCGA dataset}

We further used TCGA dataset to investigate SNHG6 expression level in human cancers. As shown in Fig. 5a, SNHG6 was upregulated in kidney chromophobe $(\mathrm{KICH})$, kidney renal clear cell carcinoma (KIRC), kidney renal papillary cell carcinoma (KIRP), glioblastoma multiforme (GBM) and colon adenocarcinoma (COAD) when compared with non-cancer tissues. The violin plot indicated that SNHG6 expression was significantly associated with clinical stage in these human cancers (Fig. 5b). Finally, a survival plot merging SNHG6 expression data and OS data of RCC, GBM and CRC from the TCGA dataset were performed. As shown in Fig. 5c, the overexpression of SNHG6 was significantly associated with an unfavorable OS, which was consistent with our results in this meta-analysis.

\section{Discussion}

In the past decades, a large amount of lncRNA transcripts were discovered by high throughput genome sequencing technologies. Along with the emerging evidence, the pivotal role of lncRNAs in oncogenesis was unveiled gradually $[28,29]$. IncRNAs, including SNHG6, were also

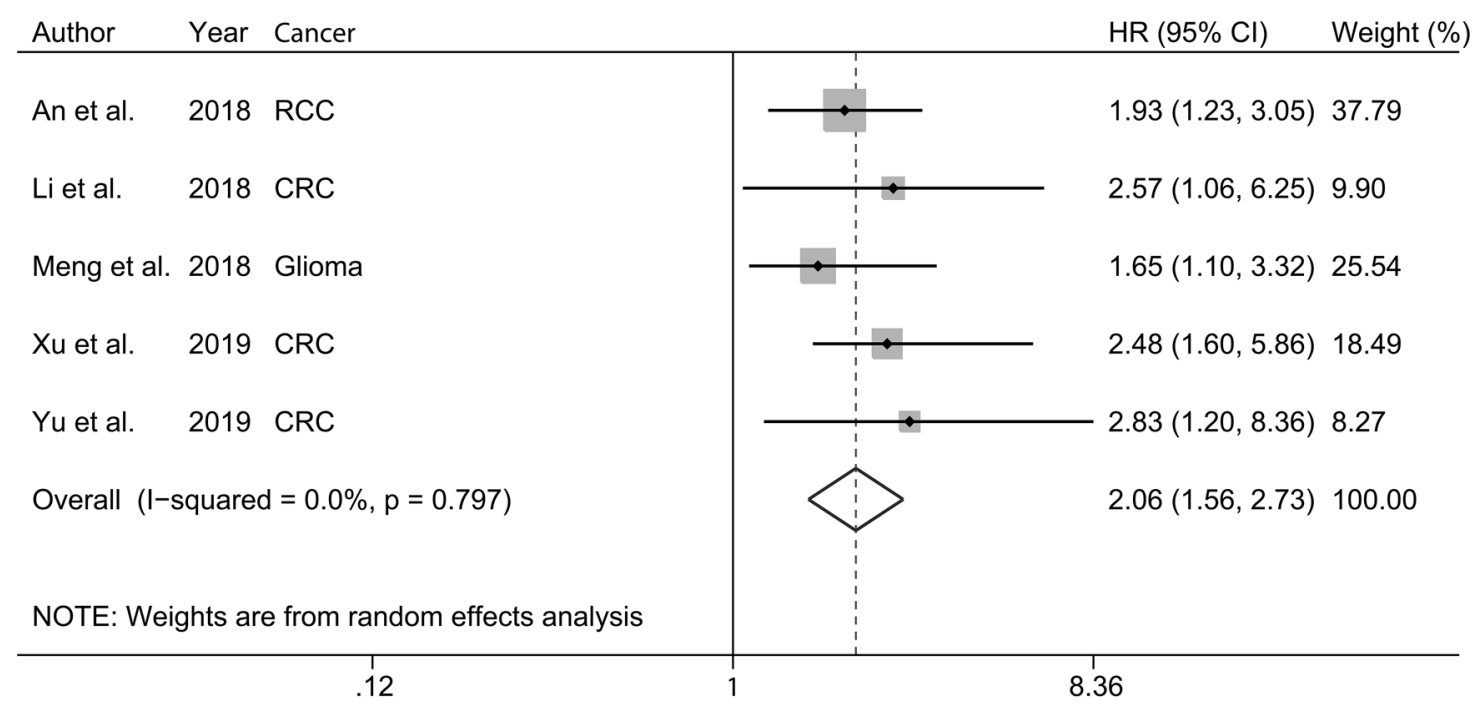

Fig. 2 Forest plot for the correlation between expression level of SNHG6 and overall survival (OS) 
Meta-analysis estimates, given named study is omitted I Lower CI Limit

OEstimate

I Upper Cl Limit

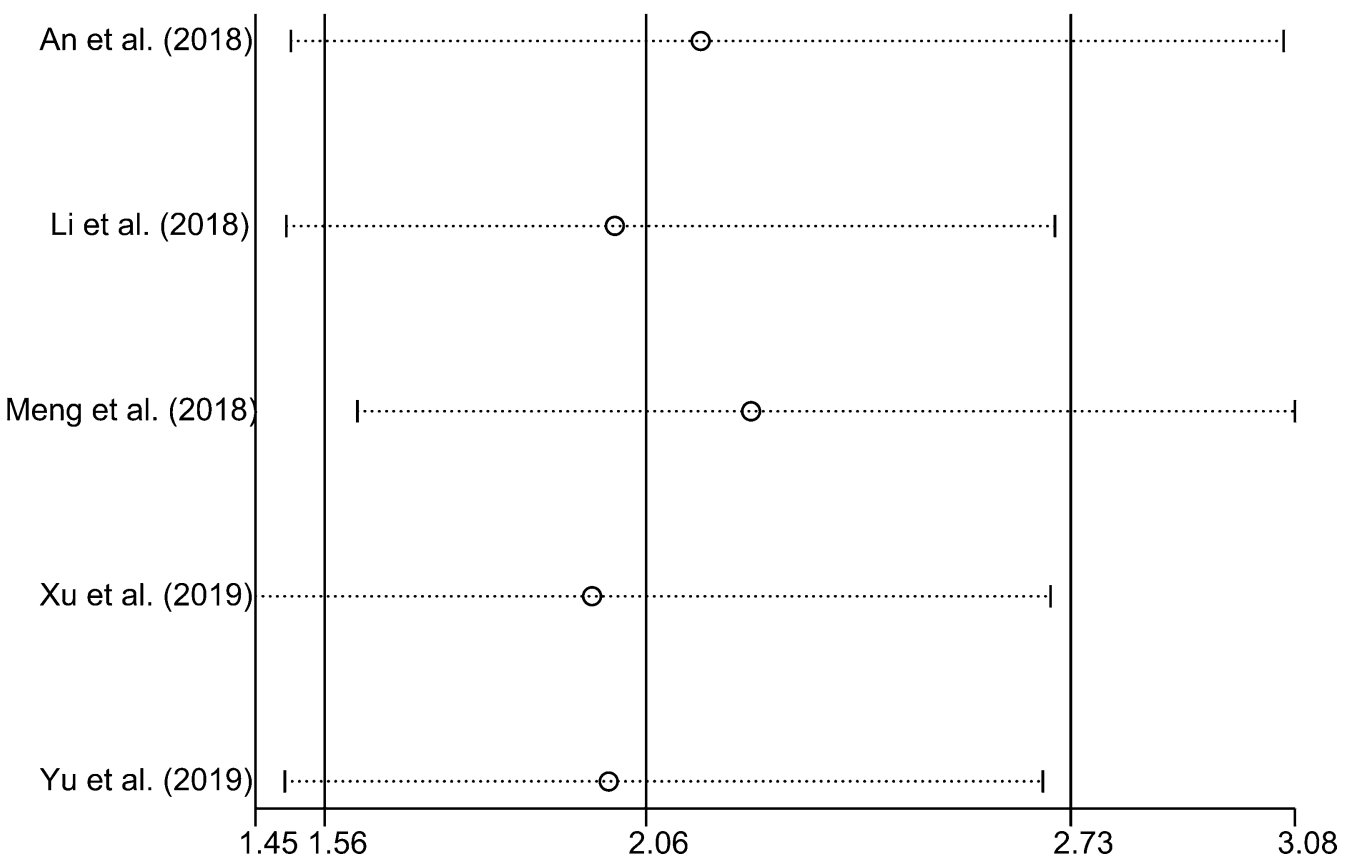

Fig. 3 Sensitivity analysis of the included studies concerning SNHG6 and overall survival (OS)

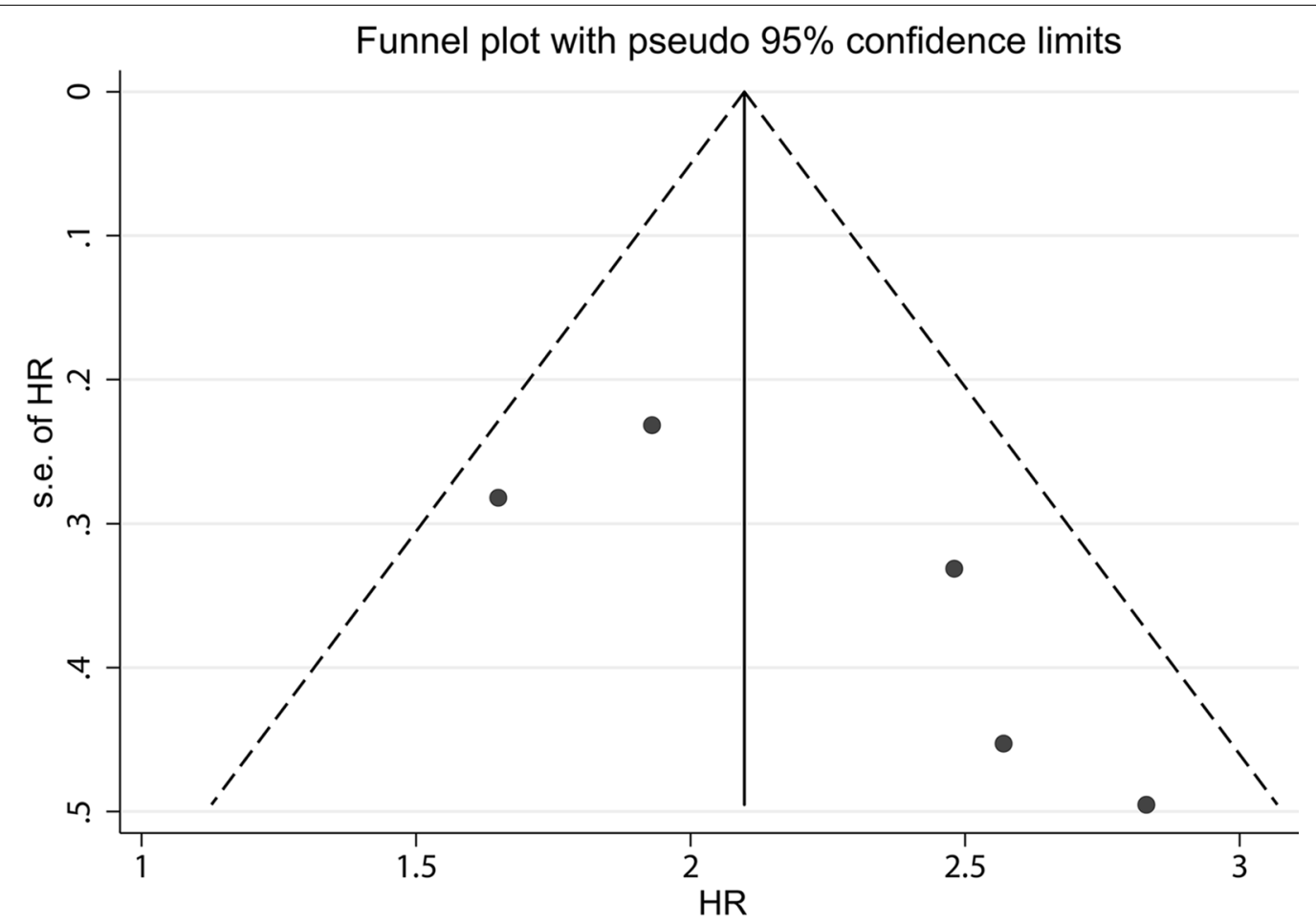

Fig. 4 Funnel plot of SNHG6 for overall survival 


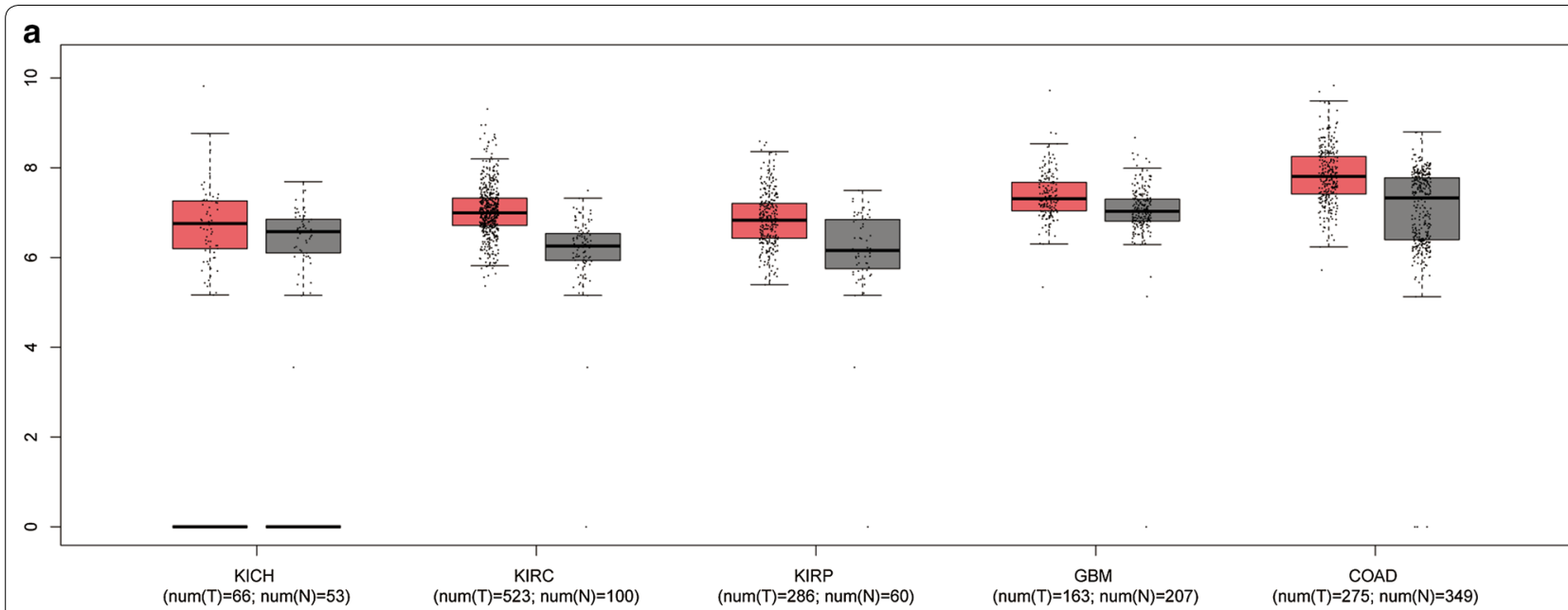

b

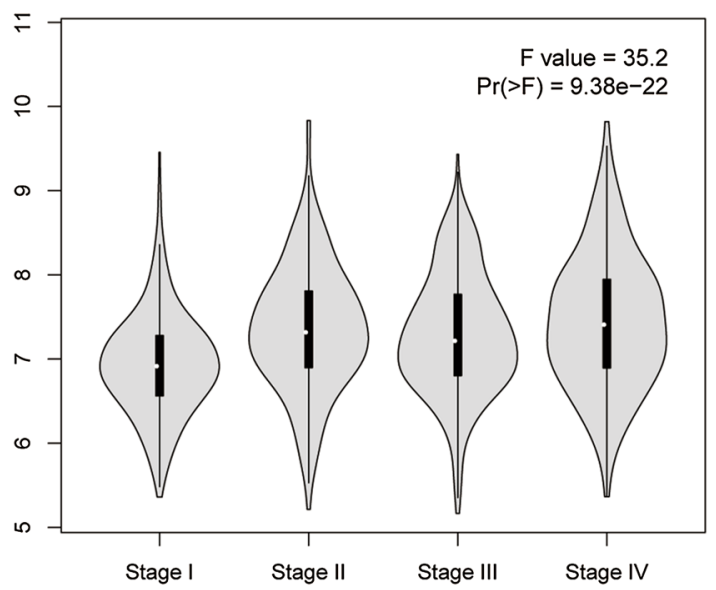

C

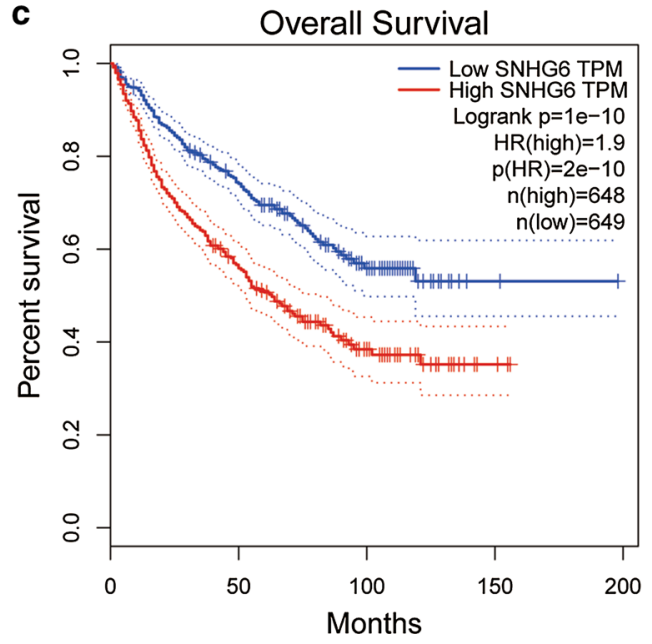

Fig. 5 Validation of SNHG6 expression of various cancers in the TCGA dataset. a The expression levels of SNHG6 in KICH, KIRC, KIRP, GBM and COAD. b Violin plot showing that SNHG6 expression was significantly associated with clinical stage in these human cancers. c Overall survival plot of SNHG16 in TCGA cohort $(n=1297$, log-rank $p<0.001)$

indicated as prognostic biomarkers in literature [23, 24]. However, the prognostic value of SNHG6 was limited and contentious due to the small sample size. Therefore, our study aimed to clarify the potential prognostic value in multiple cancers through a pooled analysis.

As a novel lncRNA, SNHG6 had been discovered overexpressed in various types of cancers, including CRC [30], HCC [16], breast cancer [31], gastric cancer [32], lung cancer [33], glioma [25] and osteosarcoma [18]. In addition, a negative correlation between SNHG6 expression and prognosis has been demonstrated in the literature [33, 34]. As for the carcinogenesis role of SNHG6, the underlying molecular mechanisms had been partly elucidated. Through the competing endogenous RNA (ceRNA) mechanism, SNHG6 can competitively sponge
miRNAs and regulate their target genes. In 2016, Chang et al. firstly reported that SNHG6 facilitated tumor growth and metastasis in hepatocellular carcinoma by competitively binding miR-101-3p to regulate ZEB1 [16]. Wang et al. found that the up-regulation of SNHG6 significantly repressed the expression of miR-125b and increased the NUAK1 expression [35]. miR-26a-5p/ ULK1 and miR-26a-5p/MAPK6 axis were also regulated by SNHG6 and participated in the development and progression of breast cancer and osteosarcoma, respectively $[18,31]$. Another study by Jafari-Oliayi et al. [34] also found that the expression of SNHG6 was significantly upregulated in primary breast cancers. SNHG6 silencing led to G1 cell cycle arrest and suppressed cell proliferation. Several signaling pathways can be activated by 
SNHG6, including the MAPK and JNK pathway in gastric cancer [32], PI3K/AKT/mTOR pathway and TGF- $\beta /$ Smad pathway in colorectal cancer [36, 37]. SNHG6 could also regulate cell cycle through interacting with crucial factors like p21 [32, 38]. In summary, elevated SNHG6 plays a vital role in cancer cell proliferation, migration and invasion.

In this meta-analysis, five original studies with a total of 487 cases were finally included and the results were pooled with adjustment for multiple confounding factors. The overall analysis showed that a high expression of SNHG6 was significantly correlated with an unfavorable OS (HR $=2.06,95 \%$ CI 1.56-2.73). Moreover, the results of subgroup analysis were also demonstrated the negative correlation between expression level of SNHG6 and prognosis, which was consistent with overall analysis.

Several limitations of our study should be acknowledged. First, because we only included studies providing multi-adjusted results, the number of papers included in final analysis was relatively small. Secondly, all of the five included studies were performed in China with Chinese population. Therefore, the generalization of our findings is relatively limited. Thirdly, various therapies for different patients may have been used in included studies, which can lead to some bias. Finally, the definition for high SNHG6 expression was obscure and might be different in the included studies, which may also affect the final pooled results.

\section{Conclusion}

In summary, the results of this meta-analysis support that SNHG6 overexpression is significantly associated with a poor prognosis in human cancers. SNHG6 may become a novel molecular target for treatment and prognostic evaluation. However, due to the limitations of our study, more well-designed studies are warranted to validate the role of SNHG6 in human cancers.

\section{Supplementary information}

Supplementary information accompanies this paper at https://doi. org/10.1186/s12935-020-01383-9.

Additional file 1: Figure S1. The mRNA expression of SNHG6 in two RCC cell lines (786-O and Caki-1) and one normal kidney cell line (HK-2) was detected by qRT-PCR.

\section{Abbreviations}

IncRNA: Long non-coding RNA; WHO: World Health Organization; HCC: Hepatocellular carcinoma; EMT: Epithelial to mesenchymal transition; HR: Hazard ratio; Cl: Confidence interval; OS: Overall survival; TCGA: The Cancer Genome Atlas; NOS: Newcastle-Ottawa Scale; CRC: Colorectal cancer; RCC : Renal cell carcinoma; KICH: Kidney chromophobe; KIRC: Kidney renal clear cell carcinoma; KIRP: Kidney renal papillary cell carcinoma; GBM: Glioblastoma multiforme; COAD: Colon adenocarcinoma; ceRNA: Competing endogenous RNA.

\section{Acknowledgements}

We thank all researchers and participants of included studies for their contributions. This study was supported by the Zhejiang Medical and Health Science and Technology Project (2020KY542) and the National Natural Science Foundation of China (81702500).

\section{Authors' contributions}

Conceived and designed the study: XX and BL. Performed the study: HXS, QWM, XX and BL. Analyzed the data: XX and QWM. Contributed analysis tools/ materials: HXS, QWM, XX and BL. Wrote the paper: HXS, QWM and XX. All authors read and approved the final manuscript.

\section{Availability of data and materials}

All data and materials analyzed in this study are included in this published article.

\section{Ethics approval and consent to participate}

Not applicable.

\section{Consent for publication}

Not applicable.

\section{Competing interests}

The authors declare no competing interests in this study.

\section{Author details}

${ }^{1}$ Department of Urology, First Affiliated Hospital, School of Medicine, Zhejiang University, Qingchun Road 79, Hangzhou 310003, Zhejiang, China. ${ }^{2}$ Department of Urology, Shengzhou People's Hospital, Shengzhou 312400, Zhejiang, China.

Received: 13 December 2019 Accepted: 26 June 2020

Published online: 06 July 2020

\section{References}

1. Binabaj MM, Bahrami A, Bahreyni A, Shafiee M, Rahmani F, Khazaei M, et al. The prognostic value of long noncoding RNA MEG3 expression in the survival of patients with cancer: a meta-analysis. J Cell Biochem. 2018;119(11):9583-90.

2. Siegel RL, Miller KD, Jemal A. Cancer statistics, 2019. CA A Cancer J Clin. 2019;69(1):7-34.

3. Djebali S, Davis CA, Merkel A, Dobin A, Lassmann T, Mortazavi A, et al. Landscape of transcription in human cells. Nature. 2012;489(7414):101-8.

4. Martens-Uzunova ES, Bottcher R, Croce CM, Jenster G, Visakorpi T, Calin GA. Long noncoding RNA in prostate, bladder, and kidney cancer. Eur Urol. 2014;65(6):1140-51.

5. Schmitt AM, Chang HY. Long Noncoding RNAs in Cancer Pathways. Cancer Cell. 2016;29(4):452-63.

6. Dunham I, Kundaje A, Aldred S, et al. An integrated encyclopedia of DNA elements in the human genome. Nature. 2012;489(7414):57-74.

7. Stein LD. Human genome: end of the beginning. Nature. 2004;431(7011):915-6.

8. Fatica A, Bozzoni I. Long non-coding RNAs: new players in cell differentiation and development. Nat Rev Genet. 2014;15(1):7-21.

9. Holoch D, Moazed D. RNA-mediated epigenetic regulation of gene expression. Nat Rev Genet. 2015;16(2):71-84.

10. Mao X, Su Z, Mookhtiar AK. Long non-coding RNA: a versatile regulator of the nuclear factor-kappaB signalling circuit. Immunology. 2017;150(4):379-88.

11. Mercer TR, Mattick JS. Structure and function of long noncoding RNAs in epigenetic regulation. Nat Struct Mol Biol. 2013;20(3):300-7.

12. Poliseno L, Salmena L, Zhang J, Carver B, Haveman WJ, Pandolfi PP. A coding-independent function of gene and pseudogene mRNAs regulates tumour biology. Nature. 2010;465(7301):1033-8.

13. Gibb EA, Brown CJ, Lam WL. The functional role of long non-coding RNA in human carcinomas. Mol Cancer. 2011;10:38.

14. Prensner JR, Chinnaiyan AM. The emergence of IncRNAs in cancer biology. Cancer Discov. 2011;1(5):391-407. 
15. Peng WX, Koirala P, Mo YY. LncRNA-mediated regulation of cell signaling in cancer. Oncogene. 2017;36(41):5661-7.

16. Chang L, Yuan Y, Li C, Guo T, Qi H, Xiao Y, et al. Upregulation of SNHG6 regulates $Z E B 1$ expression by competitively binding miR-101-3p and interacting with UPF1 in hepatocellular carcinoma. Cancer Lett. 2016;383(2):183-94.

17. Cao C, Zhang T, Zhang D, Xie L, Zou X, Lei L, et al. The long non-coding RNA, SNHG6-003, functions as a competing endogenous RNA to promote the progression of hepatocellular carcinoma. Oncogene. 2017;36(8):1112-22.

18. Zhu X, Yang G, Xu J, Zhang C. Silencing of SNHG6 induced cell autophagy by targeting miR-26a-5p/ULK1 signaling pathway in human osteosarcoma. Cancer Cell Int. 2019:19:82.

19. Moher D, Liberati A, Tetzlaff J, Altman DG, Group P. Preferred reporting items for systematic reviews and meta-analyses: the PRISMA statement BMJ. 2009;339:b2535.

20. Tang Z, Li C, Kang B, Gao G, Li C, Zhang Z. GEPIA: a web server for cancer and normal gene expression profiling and interactive analyses. Nucleic Acids Res. 2017:45(W1):W98-102.

21. DerSimonian R, Laird N. Meta-analysis in clinical trials. Control Clin Trials. 1986;7(3):177-88

22. Higgins JP, Thompson SG. Quantifying heterogeneity in a meta-analysis. Stat Med. 2002;21(11):1539-58.

23. An HX, Xu B, Wang Q, Li YS, Shen LF, Li SG. Up-regulation of long noncoding RNA SNHG6 predicts poor prognosis in renal cell carcinoma. Eur Rev Med Pharmacol Sci. 2018;22(24):8624-9.

24. Li M, Bian Z, Yao S, Zhang J, Jin G, Wang X, et al. Up-regulated expression of SNHG6 predicts poor prognosis in colorectal cancer. Pathol Res Pract. 2018;214(5):784-9.

25. Meng Q, Yang BY, Liu B, Yang JX, Sun Y. Long non-coding RNA SNHG6 promotes glioma tumorigenesis by sponging miR-101-3p. Int J Biol Mark. 2018;33(2):148-55.

26. Xu M, Chen X, Lin K, Zeng K, Liu X, Xu X, et al. IncRNA SNHG6 regulates EZH2 expression by sponging miR-26a/b and miR-214 in colorectal cancer. J Hematol Oncol. 2019;12(1):3.

27. Yu C, Sun J, Leng $X$, Yang J. Long noncoding RNA SNHG6 functions as a competing endogenous RNA by sponging miR-181a-5p to regulate E2F5 expression in colorectal cancer. Cancer Manage Res. 2019;11:611-24.

28. Pecero ML, Salvador-Bofill J, Molina-Pinelo S. Long non-coding RNAs as monitoring tools and therapeutic targets in breast cancer. Cell Oncol. 2019;42(1):1-12.
29. Calore F, Lovat F, Garofalo M. Non-coding RNAs and cancer. Int J Mol Sci. 2013;14(8):17085-110.

30. Wang X, Lan Z, He J, Lai Q, Yao X, Li Q, et al. LncRNA SNHG6 promotes chemoresistance through ULK1-induced autophagy by sponging miR26a-5p in colorectal cancer cells. Cancer Cell Int. 2019;19:234.

31. Lv P, Qiu X, Gu Y, Yang X, Xu X, Yang Y. Long non-coding RNA SNHGG enhances cell proliferation, migration and invasion by regulating miR-26a-5p/MAPK6 in breast cancer. Biomed Pharmacother. 2019:110:294-301.

32. LiY, Li D, Zhao M, Huang S, Zhang $Q$, Lin $H$, et al. Long noncoding RNA SNHG6 regulates p21 expression via activation of the JNK pathway and regulation of EZH2 in gastric cancer cells. Life Sci. 2018;208:295-304.

33. Liang $R$, Xiao G, Wang M, Li X, Li Y, Hui Z, et al. SNHG6 functions as a competing endogenous RNA to regulate E2F7 expression by sponging miR-26a-5 $p$ in lung adenocarcinoma. Biomed Pharmacother. 2018;107:1434-46.

34. Jafari-Oliayi A, Asadi MH. SNHG6 is upregulated in primary breast cancers and promotes cell cycle progression in breast cancer-derived cell lines. Cell Oncol. 2019;42(2):211-21.

35. Wang C, Tao W, Ni S, Chen Q. Upregulation of IncRNA snoRNA host gene 6 regulates NUAK family SnF1-like kinase-1 expression by competitively binding microRNA-125b and interacting with Snail $1 / 2$ in bladder cancer. J Cell Biochem. 2019;120(1):357-67.

36. Meng S, Jian Z, Yan X, Li J, Zhang R. LncRNA SNHG6 inhibits cell proliferation and metastasis by targeting ETS1 via the PI3K/AKT/mTOR pathway in colorectal cancer. Mol Med Rep. 2019;20(3):2541-8.

37. Wang X, Lai Q, He J, Li Q, Ding J, Lan Z, et al. LncRNA SNHG6 promotes proliferation, invasion and migration in colorectal cancer cells by activating TGF-beta/Smad signaling pathway via targeting UPF1 and inducing EMT via regulation of ZEB1. Int J Med Sci. 2019;16(1):51-9.

38. Ruan J, Zheng L, Hu N, Guan G, Chen J, Zhou X, et al. Long noncoding RNA SNHG6 promotes osteosarcoma cell proliferation through regulating p21 and KLF2. Arch Biochem Biophys. 2018;646:128-36.

\section{Publisher's Note}

Springer Nature remains neutral with regard to jurisdictional claims in published maps and institutional affiliations.
Ready to submit your research? Choose BMC and benefit from:

- fast, convenient online submission

- thorough peer review by experienced researchers in your field

- rapid publication on acceptance

- support for research data, including large and complex data types

- gold Open Access which fosters wider collaboration and increased citations

- maximum visibility for your research: over $100 \mathrm{M}$ website views per year

At BMC, research is always in progress.

Learn more biomedcentral.com/submissions 